\title{
EFICÁCIA ANTI-HELMÍNTICA DA IVERMECTINA “POUR-ON" COMPARADA COM A FORMULAÇÃO ORAL EM GEL NOS EQÜINOS
}

\author{
The Anthelminthic Efficiency of "Pour- On" Ivermectin \\ Compared With the Pasted Oral Formulation in Horses
}

\author{
Peterson Triches Dornbusch ${ }^{1}$ \\ Pedro Vicente Michelotto ${ }^{1}$ \\ Vamiltom Alvares Santarém ${ }^{2}$ \\ Lorena Moreira De Carli3 \\ Carlos Eduardo Camargo 3 \\ Janaina Socolovski Biava ${ }^{4}$
}

\section{Resumo}

O objetivo deste trabalho foi comparar a eficácia da ivermectina em formulação oral em gel com a formulação "pour-on" em eqüinos. Foram utilizados 24 animais, distribuídos em dois grupos $(\mathrm{n}=12)$, onde o grupo 1 recebeu ivermectina por via oral e o grupo 2 por via "pour-on". Foram realizados exames coproparasitológicos antes da administração do anti-helmíntico para se obter os valores de ovos por grama de fezes (OPG) e dividir os grupos de forma a se obter uma carga parasitária semelhante em número. Os exames coproparasitológicos foram realizados aos 7, 14, 21 e 28 dias após administração dos medicamentos. Verificou-se que o tratamento via oral apresentou redução significativa da contagem de OPG $(p<0,05)$, o que não ocorreu nos animais quando a ivermectina foi aplicada sob a forma "pour on". Foi possível concluir que a formulação existente da ivermectina "pour-on" usada em bovinos não é eficaz para eqüinos, não sendo indicada a sua utilização nessa espécie.

Palavras-chave: Ivermectina; "Pour-on"; Anti-helmínticos; Eqüinos.

1 Docente de Clínica e Cirurgia de Eqüinos - PUCPR, e-mail: p.triches@pucpr.br, endereço: Pontifícia Universidade Católica do Paraná. BR 376, km 14. Costeira. 83010-500 - São José dos Pinhais, PR - Brasil.

2 Docente do Curso de Medicina Veterinária da Unoeste/Pres. Prudente - SP, e-mail: vamilton@unoeste.br, Universidade do Oeste Paulista, Faculdade de Ciências Agrárias, Curso de Medicina Veterinária. Rodovia Raposo Tavares, km. 572. Limoeiro. 19001970 - Presidente Prudente, SP - Brasil.

3 Médicos Veterinários Graduados na PUCPR.

4 Pós-graduanda da Clínica Veterinária da FMVZ - Unesp/Botucatu - SP. Endereço: Universidade Estadual Paulista Júlio de Mesquita Filho, Faculdade de Medicina Veterinária e Zootecnia de Botucatu. Distrito de Rubião Jr., s/n. 18618-000 - Botucatu, SP - Brasil - Caixa-Postal: 560. 


\section{Abstract}

Intending to compare the efficiency of ivermectin, indicated to the bovine, pasted oral formulation with the "pour-on" in the equine, it was used 24 animals, distributed in two groups, each one of them receiving orally or "pour-on" treatment. It was made the coproparasitologic exams with feces collected on the $7^{\text {th }}, 14^{\text {th }}, 21^{\text {th }}$ and $28^{\text {th }}$ days after the application of the product. It was verified that the oral treatment showed better results, with significant reduction of the eggs of parasite on the feces $(p<0.05)$, fact that did not occur when the ivermectin was applied by "pour-on" method. It was concluded that the actual formulation of "pour-on" ivemectin used in bovine is not efficient to horses, therefore it is not indicated for that purpose.

Keywords: Ivermectin; "Pour-on"; Anthelminthics; Horses.

\section{Introdução}

Atualmente, o parasitismo é responsável por grandes perdas econômicas dentro da eqüinocultura, causando diversas enfermidades de importância clínica e sanitária (DAVIES et al., 1997; ALBERT et al., 2001). Visando a diminuir o impacto dessas afecções, o mercado oferece considerável número de fármacos eficazes e seletivos no tratamento das helmintoses (ALMEIDA; AYRES, 2002).

A descoberta do grupo das lactonas macrocíclicas, em 1975, que compreende as "avermectinas", causou grande impacto no setor agropecuário, devido à possibilidade de ser utilizado em diferentes espécies animais e pela elevada eficiência. Sua atividade endectocida depende, entre outros fatores, de sua concentração, do tempo de exposição ao parasito, da condição corpórea, da via de administração e da espécie a que é destinada (COSTA, 2004). Dentre esses fármacos, encontram-se as ivermectinas, compostos orgânicos sintéticos, que apresentam atividade sobre os estágios adultos e em desenvolvimento de nematóides gastrintestinais e pulmonares (LOVE; CHRISTLEY, 2004).

A indústria farmacêutica vem disponibilizando no mercado diversas formulações para o uso das ivermectinas, sendo mais comuns à via oral, na forma de suspensão, gel e comprimido. As formulações para aplicação via injetável e para uso tópico foram desenvolvidas recentemente e conhecida como "pour-on" (SPINOSA et al., 2002). As ivermectinas "pour-on" vêm sendo utilizadas em bovinos como um método de administração prática, com baixo poder residual, menor desperdício e estresse para o animal (LAFFONT et al., 2003). Entretanto, a cinética de absorção das drogas anti-helmínticas por meio da pele nos bovinos não está bem de- finida e nos eqüinos não foi descrita (ALMEIDA; AYRES, 2002).

O objetivo do presente trabalho foi testar a eficácia anti-helmíntica do uso da ivermectina "pour-on", utilizada para bovinos e comparar com a formulação oral, na forma gel, nos eqüinos.

\section{Material e Métodos}

Foram utilizados 24 eqüinos de raças variadas, pertencentes ao Regimento de Cavalaria da Polícia Militar do Paraná. Esses animais encontravam-se estabulados e utilizados em regime de patrulha de oito horas diárias. Eram alimentados com ração comercial, feno e pastagem cortada e fornecida no cocho. Amostras de fezes foram colhidas diretamente da ampola retal dos animais e submetidas aos exames de Gordon e Whitlock (modificada), para contagem de ovos por grama de fezes (OPG) (UENO; GONÇALVES, 1994).

Os animais foram distribuídos, aleatoriamente, mediante os resultados dos OPG, para obtenção de dois grupos $(n=12)$ com contagem total semelhante. Os animais do grupo 1 receberam tratamento com ivermectina "pour-on", na dose de $0,5 \mathrm{mg} / \mathrm{kg}$, aplicado sobre o dorso, desde a cernelha até a base da cauda, e no grupo 2 , tratamento com ivermectina via oral, em gel, na dose de $0,2 \mathrm{mg} / \mathrm{kg}$.

Amostras de fezes foram coletadas aos 7, 14, 21 e 28 dias após a administração dos produtos, sendo processadas de acordo com a técnica de flutuação descrita. Os resultados foram submetidos ao teste de análise de variância, seguida pelo teste de Bonferroni para a comparação da contagem de OPG entre os momentos. A fim de comparar a eficácia dos produtos em cada 
momento, utilizou-se o teste t de Student com correção de Welch. Para os momentos em que todos os animais apresentaram OPG igual a zero, os valores foram corrigidos para cinqüenta, a fim de possibilitar o tratamento estatístico. Os resultados foram considerados estatisticamente significativos ao nível de 5\% (TRIOLA, 1999).

\section{Resultados e discussão}

Na tabela 1 são comparadas as médias das contagens de OPG dos animais tratados com ivermectina "pour on" e ivermectina oral. Verificou-se que o tratamento com ivermectina via oral em gel apresentou os melhores resultados, com redução significativa dos valores de OPG, o que não ocorreu nos animais quando a droga foi aplicada sob a forma "pour on".

TABELA 1 - Contagem média ( \pm desvio-padrão) de ovos por grama de fezes em equiinos tratados com ivermectina pour-on (grupo 1) e com ivermectina gel (grupo 2). Comparação das médias entre momentos pela análise de variância seguida pelo teste Bonferroni, e comparação de OPG entre tratamentos pelo teste de student com relação de Welch $(p<0,05)$, Curitiba, 2004.

Table 1 - Average counting ( \pm standard deviation) of eggs per gram of equines feces, treated with ivermectin pour-on (group 1) and with ivermectin gel (group 2). Comparison of the averages between moments for the analysis of variance followed by the Bonferroni test, and comparison of OPG between treatments using the student test in relation to Welch ( $p<0.05)$, Curitiba, 2004.

\begin{tabular}{llllll}
\hline \multicolumn{5}{c}{ Dias de Colheitas } \\
\hline & $\mathbf{- 1}$ & $\mathbf{+ 7}$ & $\mathbf{+ 1 4}$ & $\mathbf{+ 2 1}$ & $\mathbf{+ 2 8}$ \\
\hline Grupo 1 & $545,83^{\mathrm{Aa}} \pm$ & $187,50^{\mathrm{Aa}} \pm$ & $229,17^{\mathrm{Aa}} \pm$ & $245,83^{\mathrm{Aa}} \pm$ & $320,83^{\mathrm{Aa}} \pm$ \\
& 806,64 & 317,75 & 242,58 & 515,87 & 371,40 \\
& & & & & $25,00^{\mathrm{Bb}} \pm$ \\
Grupo 2 & $508,33^{\mathrm{Aa}} \pm$ & $25,00^{\mathrm{Bb}} \pm$ & $25,00^{\mathrm{Bb}} \pm$ & $25,00^{\mathrm{Bb}} \pm$ \\
& 701,24 & 26,11 & 26,11 & 26,11 & 72,30 \\
\hline
\end{tabular}

- Médias seguidas de letras maiúsculas distintas representam diferença significativa entre momentos.

- Médias seguidas de letras minúsculas distintas representam diferença significativa entre os grupos em determinado momento.

Os animais que receberam tratamento com ivermectina por via oral tiveram redução inicial da carga parasitária maior, sendo que a contagem do OPG também se manteve baixa durante todo o período experimental.

A recente formulação da ivermectina na forma de "pour-on" para bovinos tem se mostrado muito eficaz pela praticidade da administração, minimizando os efeitos tóxicos nos sistemas hepático e gastrintestinal. Entretanto, neste experimento, os resultados não foram satisfatórios. Uma das possibilidades de insucesso foi a dose da ivermectina "pour-on" recomendada para bovinos, ou seja, duas vezes e meio maior que a utilizada nos animais do grupo 2, por via oral. Portanto, a necessidade de elevar a dose do medicamento deve ser estudada, mas devido aos maiores custos, o produto pode ser inviabilizado para seu uso a campo.

As diferenças da pele de cada espécie devem ser levadas também em consideração, pois o mecanismo de absorção pela pele não é bem conhecido em eqüinos, além da sudorese mais intensa, que pode levar à perda do produto pelo suor (SALOVITSZ, 2005). Laffont et al. (2003) concluíram que, mesmo em bovinos, a administração tópica não garante uma liberação controlada do fármaco.

A resistência à ivermectina não foi constatada, pois os animais do grupo 2 tiveram as contagens de OPG reduzidas a zero, dados estes também observados recentemente por outros autores que não detectaram sinais de resistência a este gru- 
po farmacológico, pelos parasitas de equinos (KAPLAN et al., 2004; MEYER, 2005). Por sua vez, Hearn; Peregrine (2003) encontraram resistência do Parascaris equorum apenas em potros tratados com ivermectina. Entretanto, os animais utilizados no experimento eram adultos, o que descarta esta possibilidade. Portanto, as ivermectinas continuam sendo altamente efetivas no tratamento e controle de uma variedade de pequenos e grandes estrongilos, bem como outras espécies de nematódeos gastrintestinais (KLEIN, 2001).

\section{Conclusões}

Com base nos resultados obtidos, concluise que diferentes formulações e vias de aplicação das ivermectinas podem causar importantes e significativas alterações na atuação e conseqüente eficácia, o que sugere a necessidade de realização de estudos farmacológicos mais detalhados sobre os compostos envolvidos. Este trabalho mostrou que a formulação existente da ivermectina "pour-on" usada em bovinos não foi eficaz para os eqüinos.

\section{Referências}

ALBERT, H. et al. Algumas considerações sobre a resistência dos parasitos aos antiparasitários e métodos de avaliação. A Hora Veterinária, Porto Alegre, v. 1, p. 36-40, 2001.

ALMEIDA, M. A. O.; AYRES, M. C. C. Considerações gerais sobre os anti-helmínticos. In. SPINOSA, H. S. et. al. Farmacologia aplicada à medicina veterinária, 3. ed. Rio de Janeiro: Guanabara Koogan, 2002. p. 459-465.

COSTA, A. J. Atividade endectocida de uma inovação quimioterápica (ivermectina + abamectina): Resultados de 12 avaliações experimentais. Revista Brasileira de Parasitologia Veteterinária, v. 13, suplemento 1, 2004.

DAVIES, J. A.; SCHWALBACH, L. M. A study to evaluate the field efficacy of ivermentin, fenbendazole and pyrantel pamoate, with preliminary observations on the efficacy of doramectin, as anthelmintics in horses. Veterinary Parasitology, v. 73, p. 243-248, 1997.
HEARN, F. P.; PEREGRINE, A. S. Identification of foals infected with Parascaris equorum apparently resistant to ivermectin. Journal American Veterinary Medical Association, v. 223, p. 482485, 2003.

KAPLAN, R. M.; KLEI, T. R.; LYONS, E. T. et al. Prevalence of anthelmintic resistant cyathostomes on horse farms. Journal American Veterinary Medical Association, v. 225, p. 903-910, 2004.

KLEIN, T. R.; S.; VISSER, M. et al. Re-evaluation of ivermectin efficacy against equine gastrointestinal parasites. Veterinary Parasitology, v. 98, p. 315320, 2001.

LAFFONT, C. M. et al. A pharmacokinetic model to document the actual disposition of topical ivermectin in cattle. Veterinary Research, v. 34, p. 445-446, 2003.

LOVE, S.; CHRISTLEY. Parasiticides. In. BERTONE, J. J.; HORSPOOL, L. J. I. Equine Clinical Pharmacology, Philadelphia: W. B. Saunders, 2004, p. 63-71.

MEYER, A.,HERTZBERG, H. Equine strongyles II. Occurrence of anthelmintic resistance in Switzerland. Schweizer Archiv fur Tierheilkunde, v. 147, p. 389-96, 2005.

SALLOVITZ, J. M.; LIFSCHITZ, A.; IMPERIALE, F. et al. Doramectin concentration profiles in the gastrointestinal tract of topically-treated calves: Influence of animal licking restriction.Veterinary Parasitology, v. 133, p. 61-70, 2005.

SPINOSA, H. S. et al. Considerações gerais sobre os anti-helmínticos. In. : ALMEIDA, M. A. O.; AYRES, M. C. C. Farmacologia aplicada à medicina veterinária. 3. ed. Rio de Janeiro, RJ: Guanabara Koogan, 2002, p. 459-465.

TRIOLA, M. F. Introdução à estatística. 7. ed. Rio de Janeiro: LTC, 1999.

UENO, H.; GONÇALVES, P. C. Manual para diagnóstico das helmintoses de ruminantes. 3. ed. Tóquio: JICA, 1994. 166 p. 